without returning to AIDS. Many of the developments outlined above are already being applied to HIV antigens. However, vaccine delivery still requires the identification of protective epitopes on antigens and the immunological effector mechanisms which generate immunity. The (relatively) small number of AIDS antigens have been dissected ad infinitum over the years. For example a relatively highly conserved sequence within the V3 loop of gp120 is one epitope which has attracted a lot of interest. However, this conference saw the report of CD8 cytotoxic Tlymphocyte directed against the Nef gene product which is conserved, produced early during infection and may even be expressed in latently infected cells. It is known that cytotoxic $\mathrm{T}$-cells have a suppressive effect on HIV infection of lymphocytes, and it is tempting to speculate that Nef may be a target for suppressive CTLs. If so, the identification of ISCOMS as carriers capable of generating a CTL response and the development of a BCG delivery system may assume particular significance.

ROBERT RIDLEY Institute of Cell and Molecular Biology University of Edinburgh Edinburgh EH9 3JR

Genetic Monitoring of Inbred Strains of Rats: a Manual on Colony Management, Basic Monitoring Techniques, and Genetic Variants of the Laboratory Rat. Edited by HaNS J. HedRICH. Gustav Fischer Verlag, Stuttgart and New York. 1990. 539 pages. Hardback. DM 198. ISBN 3437306200.

The laboratory rat has been making an increasingly important contribution recently both to mammalian genetics and to biomedical research, the latter instanced by the fact that almost all vital organs of the rat can now be transplanted. A major problem for the research worker, particularly in biomedicine, has been the difficulty in extracting the needed information on rat genes, rat strains and inbred lines from the scattered literature; and the aim of the book under review was to fill this need and also to include full details of colony management and laboratory techniques. The editor and other contributors have succeeded admirably in this task, and their book will be of great interest to a variety of geneticists as well as helping those in applied research to improve their experimental techniques, in many cases dramatically: the reason for this is that choice of the most suitable strains and detailed knowledge of their characteristics are essential for obtaining convincing results.

The first half of the book discusses the use of inbred strains in biomedical research, genetic monitoring and colony management, types of marker gene available, and laboratory methods for identifying the alleles of polymorphic loci and other genetic variants. The marker genes include immunological markers, MHC and non-MHC alloantigenic cell-surface markers, biochemical, cytogenetic and DNA markers, and morphological and physiological traits. The chapter on laboratory methods gives detailed protocols for identifying variants in each of these classes of marker; for example, photographs and schematic interpretations of electropherograms are given for each of the 42 biochemical marker systems available. This chapter ends with an extensive discussion of the important technique of cryopreserving rat embryos and setting up an embryo bank.

The second half of the book (chapters 5 and 6) begins with a catalogue of mutant genes and polymorphic loci of the rat-covering about 330 loci of which some 200 have been mapped to linkage groups or chromosomes, compared with over 1300 loci reported and nearly 1000 of them mapped on chromosomes in the mouse. The rat gene catalogue is arranged alphabetically by locus symbol and gives detailed information about each gene: its known or probable function or effect, the alleles and their effects, and references. This arrangement sensibly mimics that for mouse genes in the new edition of Genetic Variants and Strains of the Laboratory Mouse, making homology comparisons easier, subject to historical differences in choice of names. As an example, I could not find the equivalent of any of the rat hooded alleles in the mouse catalogue - there is a mention of the piebald mouse in the discussion of hooded, but I was unable to run down a piebald locus or allele of the mouse. I suspect comparisons of this sort will become quite popular, particularly as gene mapping in the rat has increased recently using somatic cell hybridization.

The 116 pages of the rat gene catalogue are followed by a linkage map, a catalogue of inbred strains of rats giving colour, number of generations inbred, origin, characteristics (e.g. very docile, or aggressive towards handlers), etc., and holders, as far as all these are known. Some 140 inbred strains are described, of which 29 have no known holder; but it is hoped that holders of some of these strains will own up on seeing the book (or reading this review).

The book ends with some very useful tables, detailing Congenic and Segregating Inbred Strains, Recombinant Inbred Strains, Mutant-bearing Strains and Stocks, and Strain Distribution of Polymorphic Loci, with, finally, a roster of abbreviations and an index. One should also mention the fine set of colour photographs of different rat genotypes on pages 62 and 63 .

This book obviously makes a very important contribution to mammalian genetics, and should find a place on the shelves of all biological libraries and in many laboratories. Rat genetics can now be said to have come of age.

An intriguing point is raised by Fig. 3.3.7 on page 56 , which compares the lengths of the $Y$ chromosome (standardized against the length of chromosome 20) in 18 rat strains. Clearly there are large and doubtless 
significant differences in length between some of these Ys, and Kodama \& Sasaki (1977) found that, when the 66 pairs of strains which could be made from the twelve strains they measured were tested statistically, 39 pairs were significantly different from each other. This is an odd statistic which is difficult to make sense of, and a proper analysis of the data would be of interest. I deduce from simple assumptions that the number of strains with $Y$ chromosomes of significantly different lengths among these twelve strains is more than two, very probably three and just possibly four. Genetical Research $(1991,57,195)$ has recently published details of the first reported deletion in the mouse $Y$ chromosome, which causes a marked reduction in length. Can any of the rat Ys carry deletions or additions?

ERIC REEVE

Institute of Animal Genetics University of Edinburgh

\section{Mammals from Pouches and Eggs: Genetics, Breeding and Evolution of Marsupials and Monotremes. Edited by Jennifer A. Marshall Graves, Rory M. Hope and Desmond W. CoOPER. CSIRO Publications, PO Box 89, 314 Gilbert Street, East Melbourne, Victoria 3002, Australia. 1990. 337 pages. Hardback US \$50.00. ISBN 0643050205 .}

As an undergraduate in the late 1950s I gained the impression that monotremes and marsupials were interesting experiments of Nature, but nevertheless 'failed' mammals - ones that did not make the evolutionary ascent to the eutherian pinnacle. Since then, with the pioneering investigations of Sharman followed by those of Tyndale-Biscoe, Renfree and an ever-increasing number of others, numerous long-held misconceptions are being redressed: we have learned to regard the marsupials not as 'inferior' but rather as 'alternative' mammals - ones that have substituted investment in lactation for gestation as a reproductive strategy.

Marsupial research has accelerated enormously in the last two decades. With the recent introduction of the South American opossum, Monodelphis domestica, as a biomedical model, numerous laboratories outside Australia (including the U.K.) are also engaged in this pursuit. These considerations together with the current interest in sex determination and conservation, and the general lack of information available on the genetics of non-eutherian mammals, makes the publication of Mammals from Pouches and Eggs most timely.

This volume brings together topics discussed at a Boden Research Conference held in Australia in 1988 and subsequently published in the Australian Journal of Zoology (vol. 37, 1990).

The stated aim of the editors is to make available information on marsupials and monotremes to 'anyone in the field, entering the field or simply curious' and in this they succeed admirably. The book contains a wealth of information on breeding and conservation, genetics and cytogenetics, reproduction, sex determination and evolution contained in 25 lucidly written chapters, each of them well referenced. However, only a single chapter is devoted exclusively to Monotremes.

The text is grouped into four sections: I. Genetics and Reproduction; II. Immunological and Molecular Approaches to Marsupial Phylogeny; III. Chromosomes and Gene Maps of Marsupials and Monotremes; and IV. Chromosome inactivation and Sex Determination in Marsupials.

The comparative approach is maintained throughout, and much insight is to be gained thereby. Take the search for the sex-determining gene: three years ago ZFY on the eutherian Y chromosome was a putative candidate, until it was found that in marsupials its homologue mapped to an autosome; this immediately threw doubt on the sex-determining function of ZFY.

Comparative studies also suggest that $\mathrm{X}$ chromosome inactivation has arisen in a stepwise fashion during the course of evolution, since monotremes show incomplete, tissue-specific $\mathrm{X}$-inactivation; marsupials (and the primitive endoderm of eutherians) are characterized by incomplete paternal $\mathrm{X}$-inactivation, while eutherian embryos show random and incomplete $\mathrm{X}$-inactivation.

As a developmental biologist I found the chapters on sex determination the most intriguing. Since the pioneering work of Jost in the 1950s, mammalian sex determination has been considered as involving transformation of the indifferent gonad into a testis by the Y chromosome, followed by testosterone secretion, which then brought about masculinization of the rest of the reproductive system. In the absence of a $\mathrm{Y}$ chromosome a female would develop, by default. In marsupials, however, scrotum, pouch and mammary gland development are under primary genetic control and make their appearance prior to gonadal sex differentiation. The search is now on for sexual dimorphisms in eutherians that precede gonadal differentiation, and Shaw et al. quote several examples of this from the recent literature. As these authors state, 'studies in marsupials are alerting us to the fact that the hormonal control of sexual differentiation overlies a more fundamental genetic control system'.

The homologies of pouch and scrotum have long been debated. Based on their studies of intersex marsupials Sharman et al. postulate a locus on the X chromosome which in single dose would induce scrotum development and in double dose pouch and mammary glands. They show a fascinating photo of an XXY kangaroo with penis and pouch but no scrotum. However, the recent description of mammary rudiments in normal male Monodelphis domestica suggest the hypothesis is inadequate.

A few minor errors only were noted: the misspelling Monodelphis domesticus (p. 7) and Razzin \& Riggs (p. 